NSF-ITP-98-127

hep-th/9812159

\title{
Supergravity Solutions for Localized Intersections of Branes
}

\author{
Akikazu Hashimoto \\ Institute for Theoretical Physics \\ University of California \\ Santa Barbara, CA 93106
}

\begin{abstract}
We construct an explicit supergravity solution for a configuration of localized D4brane ending on a D6-brane, restricted to the near horizon region of the latter. We generate this solution by dimensionally reducing the supergravity solution for a flat M5-brane in $R^{1,7} \times C^{2} / Z_{N}$ with the M5-brane partially embedded in $C^{2} / Z_{N}$. We describe the general class of localized intersections and overlaps whose supergravity solutions are constructible in this way.
\end{abstract}

December 1998 


\section{Introduction}

Much of the recent advances in string theory are derived from the properties of brane objects which exist in the theory. Originally, these objects were formulated as a $p$-brane solution [1] to the supergravity field equations which are the low-energy effective description of string theory. The solutions described in [1] were flat along the world-volume and spherically symmetric in the directions transverse to the brane. A possibility to study these objects microscopically was opened following the realization that certain class of these branes are D-branes, a type of topological defect on which a string can end [2]. Even branes which are not D-branes can in general be thought of as their duals.

From the perspective of string theory, it is easy and instructive to consider a more complicated configuration such as localized (as opposed to smeared) intersections and junctions of strings. Wide range of applications of these brane configurations have been discussed by many authors, including such topics as the brane construction of gauge theories [3], BIons [4, 5], string junctions and networks [6, 7, 8], just to name a few. Related configurations for topological defects in ordinary field theories have been discussed in $[9]$.

It is natural to wonder the extent to which phenomena associated with these nontrivial brane configurations could have been discussed in the original formulation of branes as solutions to the supergravity equations of motion. This question is of acute interest especially in light of the recent realization that supergravity provides an effective dual description to strongly coupled large $N$ gauge dynamics [10]. Unfortunately, progress in this line of investigation is obstructed by our lack of knowledge of the explicit supergravity solutions corresponding to these backgrounds. Localized intersections break the spherical symmetry along the the transverse directions, and techniques for solving supergravity field equations in such a general context is not yet known. Indeed, explicit form of the solutions are known only for the few special cases $[11,12,13,14,15]$.

Recently, interest have focused on the near horizon geometry of branes following the work of [10]. By restricting one's attention to the near horizon geometry, the problem of finding supergravity background corresponding to localized intersections also simplify. Explicit expression for D-instanton localized in the near horizon $\mathrm{AdS}_{5}$ geometry of the D3-brane and its relation to gauge theory instantons were discussed in $[16,17]$.

In a related but independent line of development, the authors of [18] have shown that a certain localized brane solution can be derived explicitly in the near horizon region of the D6-brane. The main observation behind [18] is the fact that the near horizon geometry of 
$N$ D6-brane in type IIA supergravity admits an interpretation as a dimensional reduction of 11-dimensional supergravity on $R^{1,6} \times \Sigma$ where $\Sigma$ is an ALE space $C^{2} / Z_{N}$ [19]. It's universal cover is therefore the flat 11-dimensional Minkowski space. The authors of [18] then considers the supergravity solution of the flat M2 and M5 branes embedded in the $R^{1,6}$ part of the universal cover. By moding the action of the $Z_{N}$ group and reducing to type IIA, they constructed localized supergravity solutions of D2 and NS5-branes in the near horizon region of the D6-brane, as well as other configurations related by U-duality.

The main goal of this paper is to point out that it is possible to partially (or entirely) embed the M2 and M5 into the ALE part of the space in such a way that these branes are flat in the universal cover. Therefore, by following the same steps of orbifolding and dimensionally reducing, one can obtain an explicit expression for a new class of localized brane geometry in the near horizon region of the D6-brane. Although these solutions are far from being general, such an explicit solution might serve as a useful aid in the on-going pursuit for a general construction of supergravity solutions for localized intersections.

This paper is organized as follows. We begin in section 2 by reviewing the basic construction of the near horizon D6-brane geometry as a dimensional reduction of an ALE orbifold, and present an explicit construction for the space-time background of a localized configuration of D4-brane ending on a near-horizon core of the D6-brane. In section 3, we explore a general class of brane configurations which can be obtained using this method and present few more examples, including the overlapping NS5 and D6-branes. Comments and conclusions are collected in section 4.

\section{D4-brane ending on D6-brane}

We begin this section by briefly reviewing the basic construction of the near horizon geometry of the D6-brane as a dimensional reduction of an ALE orbifold. This was essentially worked out in $[18,19]$ but we repeat it here for completeness and to fix the notation.

Let us begin by considering 11-dimensional supergravity on $R^{1,6} \times \Sigma$ where $\Sigma$ is an ALE space of type $A_{N-1}$. For our purposes, it is convenient to think of this space as a $Z_{N}$ orbifold of $(v, w) \in C^{2}$ under the action $(v, w) \rightarrow\left(e^{2 \pi i / N} v, e^{2 \pi i / N} w\right)$. By setting $v=x_{8}+i x_{9}$ and $w=x_{7}+i x_{10}$, we can think of $R^{1,6} \times \Sigma$ as $R^{1,10} / Z_{N}$ with $Z_{N}$ acting on $(7,8,9,10)$ coordinates. 
The metric on $R^{1,10}$ is simply

$$
d s_{11}^{2}=-d x_{0}^{2}+\sum_{i=1}^{10} d x_{i}^{2} .
$$

To make the $Z_{N}$ action on this background transparent, it is convenient to change variables

$$
w=x_{7}+i x_{10}=\rho e^{i \tilde{\phi}} \cos (\tilde{\theta}), \quad v=x_{8}+i x_{9}=\rho e^{i \tilde{\psi}} \sin (\tilde{\theta})
$$

with ranges $0 \leq \tilde{\theta} \leq \pi / 2,0 \leq \tilde{\phi}, \tilde{\psi} \leq 2 \pi$, and $0 \leq \rho$. Orbifolding by $Z_{N}$ instructs us to identify $(\tilde{\phi}, \tilde{\psi}) \sim(\tilde{\phi}, \tilde{\psi})+(2 \pi / N, 2 \pi / N)$. In terms of these variables, the metric takes the form

$$
d s_{11}^{2}=d x_{\|}^{2}+d \rho^{2}+\rho^{2}\left(d \tilde{\theta}^{2}+\sin ^{2}(\tilde{\theta}) d \tilde{\psi}^{2}+\cos ^{2}(\tilde{\theta}) d \tilde{\phi}^{2}\right) .
$$

Further change of variables

$$
U=\frac{\rho^{2}}{2 N l_{p}^{3}}, \quad \theta=2 \tilde{\theta}, \quad \psi=\tilde{\psi}-\tilde{\phi}, \quad x_{11}=R_{11} \phi=R_{11} N \tilde{\phi}
$$

leads to a metric of the form

$$
d s_{11}^{2}=d x_{\|}^{2}+\frac{l_{p}^{3} N}{2 U} d U^{2}+\frac{l_{p}^{3} N U}{2}\left(d \theta+\sin ^{2}(\theta) d \psi\right)+\frac{2 U l_{p}^{3}}{N R_{11}}\left[d x_{11}+\frac{N R_{11}}{2}(\cos (\theta)-1) d \psi\right]^{2}
$$

where $x_{11}$ is periodic under shift by $2 \pi R_{11}$. Dimensionally reducing along $x_{11}$ leads to a type IIA metric of the form

$$
\begin{aligned}
d s_{10}^{2} & =\alpha^{\prime}\left[\frac{(2 \pi)^{2}}{g_{Y M}} \sqrt{\frac{2 U}{N}} d x_{\|}^{2}+\frac{g_{Y M}}{(2 \pi)^{2}} \sqrt{\frac{N}{2 U}} d U^{2}+\frac{g_{Y M}}{(2 \pi)^{2} \sqrt{2}} \sqrt{N} U^{3 / 2} d \Omega_{2}^{2}\right], \\
e^{\phi} & =\frac{g_{Y M}^{2}}{2 \pi}\left(\frac{2 U}{g_{Y M}^{2} N}\right)^{3 / 4}, \\
A^{(1)} & =\frac{N R_{11}}{2}(\cos (\theta)-1) d \psi,
\end{aligned}
$$

where $d \Omega_{2}^{2}=r^{2}\left(d \theta^{2}+\sin ^{2}(\theta) d \psi^{2}\right)$. This is precisely the type IIA supergravity background for $N$ D6-branes [1] in the near horizon limit [19]

$$
U=\frac{|x|}{\alpha^{\prime}}=\text { fixed, } \quad g_{Y M}^{2}=(2 \pi)^{p-2} g_{s t} l_{s}^{p-3}=\text { fixed }, \quad p=6, \quad \alpha^{\prime} \rightarrow 0 .
$$

The authors of [18] pointed out that a similar procedure can be carried out starting with a background of $n$ M2-brane extended along the $(0,1,2)$ direction and embedded anywhere in the $(3,4,5,6)$ direction and into the origin of the $(7,8,9,10)$ coordinates of $R^{1,10}$. The supergravity solution for such a background takes a simple form

$d s_{11}^{2}=f^{-2 / 3}\left(-d x_{0}^{2}+d x_{1}^{2}+d x_{2}^{2}\right)+f^{1 / 3}\left(d x_{3}^{2}+d x_{4}^{2}+d x_{5}^{2}+d x_{6}^{2}+d x_{7}^{2}+d x_{8}^{2}+d x_{9}^{2}+d x_{10}^{2}\right)$ 


$$
A^{(3)}=\frac{f-1}{f} d x_{0} \wedge d x_{1} \wedge d x_{2}
$$

and is invariant under the action of $Z_{N}$. Here, $f$ is the standard harmonic function for the M2-brane. One can therefore orbifold and dimensionally reduce along the same coordinates as in the pure ALE case. Since the dimensional reduction maps M2 brane into D2 brane, one obtains by this construction a supergravity solution for D2 brane localized along $(3,4,5,6)$ coordinates in the near horizon geometry of the D6-brane. Similar procedure using the M5 brane leads to a construction of NS5-brane localized within D6branes.

The main point of this paper is to show that a new class of brane configuration can be generated using the same technique as [18] but letting M-branes partially (or entirely) wrap $\Sigma$. As long as such embeddings arise from orbifolding a configuration of flat Mbranes in $R^{1,10}$, one can orbifold and dimensionally reduce the M-brane supergravity solution. In the remainder of this section, we will discuss one concrete example in detail, the configuration of D4-brane ending on a D6-brane. Generalizations will be discussed in the following section.

D4-branes arise from double dimensional reduction of M5-brane, so let us consider a configuration of M5-brane in $R^{1,10}$ oriented along the $(0,1,2,3,7,10)$ direction, localized anywhere along the $(4,5,6)$ direction, and localized at the origin along the $(8,9)$ direction. The $Z_{N}$ orbifold group acts on complex coordinate $w=x_{7}+i x_{10}$ and $v=x_{8}+i x_{9}$, so such a configuration is invariant under the action of $Z_{N}$. The idea is simply to take the metric of M5-brane in $R^{1,10}$

$$
\begin{gathered}
d s_{11}=f^{-1 / 3}\left(-d x_{0}^{2}+d x_{1}^{2}+d x_{2}^{2}+d x_{3}^{2}+d x_{7}^{2}+d x_{10}^{2}\right)+f^{2 / 3}\left(d x_{4}^{2}+d x_{5}^{2}+d x_{6}^{2}+d x_{8}^{2}+d x_{9}^{2}\right) \\
A_{6}=\frac{f-1}{f} d x_{0} \wedge d x_{1} \wedge d x_{2} \wedge d x_{3} \wedge d x_{7} \wedge d x_{10}
\end{gathered}
$$

where $A^{(6)}$ is the Poincare dual of the 3-form of the 11-dimensional supergravity, and perform the same manipulation performed in the above. Since all this is quite straightforward, we simply quote the result here:

$$
\begin{aligned}
d s^{2}= & \alpha^{\prime}\left[\frac{(2 \pi)^{2}}{g_{Y M}} \sqrt{\frac{2 U}{N}}\left(\Delta^{1 / 2}\left(\sum_{i=0}^{3} d x_{i}^{2}+\sum_{i=4}^{6} f d x_{i}^{2}\right)\right)+\frac{g_{Y M}}{(2 \pi)^{2}} \sqrt{\frac{N}{2 U}} f \Delta^{3 / 2} d U^{2}\right. \\
& \left.+\frac{g_{Y M}}{(2 \pi)^{2}} \sqrt{\frac{N}{2}} U^{3 / 2}\left(f \Delta^{1 / 2} \tilde{\Delta} d \theta^{2}+\Delta^{-1 / 2} \sin (\theta)^{2} d \psi^{2}+\frac{f-1}{U} \Delta^{1 / 2} \sin (\theta) d U d \theta\right)\right] \\
A^{(1)}= & \frac{\Delta^{-1} N R_{11}}{2}(1-\cos (\theta)) d \psi
\end{aligned}
$$




$$
\begin{aligned}
A^{(5)} & =\left(\frac{f-1}{f}\right) d x_{0} \wedge d x_{1} \wedge d x_{2} \wedge d x_{3} \wedge\left(\frac{l_{p}^{3}(1+\cos (\theta))}{4 R_{11}} d U-\frac{l_{p}^{3} U \sin (\theta)}{4 R_{11}} d \theta\right) \\
e^{\phi} & =\sqrt{f}\left(\frac{g_{Y M}^{2} \Delta^{3} U^{3}}{2 N^{3} \pi^{4}}\right)^{1 / 4}
\end{aligned}
$$

where

$$
\begin{gathered}
\Delta=\frac{1+f+(1-f) \cos (\theta)}{2 f}, \quad \tilde{\Delta}=\frac{1+f-(1-f) \cos (\theta)}{2 f}, \\
f=1+\frac{\pi n l_{p}^{3}}{r^{3}}, \quad r^{2}=\sum_{i=4}^{6} x_{i}^{2}+2 N l_{p}^{3} U \sin ^{2}(\theta / 2) .
\end{gathered}
$$

To derive $A^{(5)}$, we used the fact that

$$
d x_{i}=\frac{d x_{i}}{d U} d U+\frac{d x_{i}}{d \theta} d \theta+\frac{d x_{i}}{d \psi} d \psi+\frac{d x_{i}}{d x_{11}} d x_{11}
$$

This, therefore, is the metric for the localized intersection of a D4-brane and a D6-brane. A useful check is to see that the above expression simplifies to that of pure D6-brane near horizon geometry upon setting $f=1$. Note that the D4-brane is ending on instead of piercing through the D6-brane, as can be seen from the geometry of the $r=0$ locus. To emphasize the point that this is a classical supergravity solution, all dependence on $l_{s}, l_{p}$, and $g_{s t}$ can be scaled into (recalling $g_{Y M}^{2}=(2 \pi)^{p-2} g_{s t} t_{s}^{p-3}$ )

$$
y=\alpha^{\prime} U, \quad Y=\frac{g_{s t} N l_{s}}{2}, \quad R^{3}=\pi n l_{p}^{3} .
$$

In these variables, the background becomes

$$
\begin{aligned}
d s^{2}= & \sqrt{\frac{y}{Y}}\left(\Delta^{1 / 2}\left(\sum_{i=0}^{3} d x_{i}^{2}+\sum_{i=4}^{6} f d x_{i}^{2}\right)\right)+\sqrt{\frac{Y}{y} f \Delta^{3 / 2} d y^{2}} \\
& y^{3 / 2} \sqrt{Y}\left(f \Delta^{1 / 2} \tilde{\Delta} d \theta^{2}+\Delta^{-1 / 2} \sin (\theta)^{2} d \psi^{2}+\frac{(f-1)}{y} \Delta^{1 / 2} \sin (\theta) d y d \theta\right) \\
A^{(1)}= & \Delta^{-1} Y(1-\cos (\theta)) d \psi \\
A^{(5)}= & \left(\frac{f-1}{f}\right) d x_{0} \wedge d x_{1} \wedge d x_{2} \wedge d x_{3} \wedge\left(\frac{1+\cos (\theta)}{4} d y-\frac{y \sin (\theta)}{4} d \theta\right) \\
\frac{1}{g_{s t}} e^{\phi}= & \sqrt{f}\left(\frac{y}{Y} \Delta\right)^{3 / 4}
\end{aligned}
$$

with

$$
f=1+\frac{R^{3}}{r^{3}}, \quad r^{2}=\sum_{i=4}^{6} x_{i}^{2}+4 y Y \sin ^{2}(\theta / 2) .
$$




\section{Some generalizations}

In the previous section, we discussed one concrete example of spacetime background generated by orbifolding and dimensionally reducing a configuration of flat M-brane in $R^{1,10}$. In this section, we will describe the scope to which this construction can be generalized, and present some examples. In M-theory, we have M2 and M5-branes to serve as our basic building blocks. One can in principle wrap any part of the M2 or M5-brane world volume in the $R^{1,6}$ part of the target space and all the rest in the $\Sigma$. The embedding into $\Sigma$ must be such that enough supersymmetry is preserved to ensure the stability of the configuration. The issue of supersymmetry is easier to address in the case where the number of dimensions embedded into $\Sigma$ is even, where the allowed embedding geometries are holomorphic curves. The possibility therefore is to embed none, two, or four of the world volume coordinates of the M-branes into $\Sigma$. Embedding none of the coordinates was the case discussed in [19]. Only M5-brane can be used to embed four of the world volume coordinates into $\Sigma$, and this leads simply to a configuration of D4-brane and D6-brane sharing one space and one time coordinates, and relatively transverse in the remaining eight coordinates. The example described in the previous section falls in the category of embedding two of the world volume coordinates into $\Sigma$. In the remainder of this section, we will elaborate on this possibility and present further examples of supergravity backgrounds for brane intersections. We will focus mainly on embedding M5-branes although it is straightforward to extend these results to M2-branes.

We are interested in finding a holomorphic embedding of complex plane into $\Sigma$. Similar holomorphic embedding of two the world volume coordinates of the M5 brane into a Taub-NUT space was discussed in [20]. These authors studied the M-theory origin of the Hanany-Witten transition between D6 and NS5-branes [21] by considering a generic holomorphic embeddings in Taub-NUT space and studying its limiting behavior as the radius of 11-th circle at infinity is sent to zero. For our discussion of constructing supergravity solutions, however, we must restrict ourself to the near horizon ALE geometry, since the fact that the M-theory lift is an orbifold of flat space is crucial to our construction. Furthermore, we are restricted to flat embeddings.

The flat curve describing the embedding of the previous section was simply $v=0$. In general, flat embedding in $C^{2}$ is given by a linear curve $v-a w=b$, where $a$ and $b$ are complex parameters. We are further restricted by the requirement that our brane configuration be invariant under the action of the orbifold group which sends $(v, w)$ to $\left(e^{2 \pi i / N} v, e^{2 \pi i / N} w\right)$. This requirement can be satisfied by adding images, or equivalently, 
by taking

$$
(v-a w)^{N}=b^{N}
$$

as our embedding curve. In the remainder of this section we will describe the geometry and the supergravity background for this brane configuration.

\subsection{Geometry of the brane}

Let us first describe the geometry of this brane configuration from the point of view of type IIA near horizon D6-brane point of view. The embedding (3.1) turns out to describe a paraboloid oriented along the axis

$$
a=\tan (\theta / 2) e^{i \psi}
$$

with the focus located at the origin. This is demonstrated by taking the norm of (3.1)

$$
|v-a w|=|b|
$$

and applying a chain of change of variables. Let us assume without loss in generality that

$a$ is real. The specific change of variables are as follows. First apply (2.1) and (2.2) to express (3.2) in terms variables $U, \theta, \psi$, and $x_{11}$. Then go to Cartesian coordinates by defining

$$
U_{1}=U \cos (\theta), \quad U_{2}+i U_{3}=U \sin (\theta) e^{i \psi} .
$$

Finally, rotate the coordinate system by defining

$$
\tilde{U}_{1}=\frac{a^{2}-1}{1+a^{2}} U_{1}+\frac{2 a}{1+a^{2}} U_{2}, \quad \tilde{U}_{2}=-\frac{2 a}{1+a^{2}} U_{1}+\frac{a^{2}-1}{1+a^{2}} U_{2}, \quad \tilde{U}_{3}=U_{3} .
$$

In terms of these new variables (3.2) becomes

$$
\tilde{U}_{1}=-\frac{b^{2}}{2\left(1+a^{2}\right) l_{p}^{3} N}+\frac{\left(1+a^{2}\right) l_{p}^{3} N}{2 b^{2}}\left(\tilde{U}_{2}^{2}+\tilde{U}_{3}^{2}\right)
$$

which shows that this is indeed a paraboloid. We are also interested in how this paraboloid is embedded in the $x_{11}$ coordinate. A convenient way to address this issue is to parameterize the paraboloid by $z=v / w$ which amounts to labeling the points on the paraboloid by its intersection with a ray from the origin oriented along $z=\tan (\theta / 2) e^{i \psi}$. In terms of $z,(3.1)$ is simply

$$
w^{N}=\frac{b^{N}}{(z-a)^{N}} .
$$

The analytic structure of this embedding is now very clear. There is a pole at $z=a$ and a zero at $z=\infty$. This means that $w$ winds once around the complex plain if one winds 

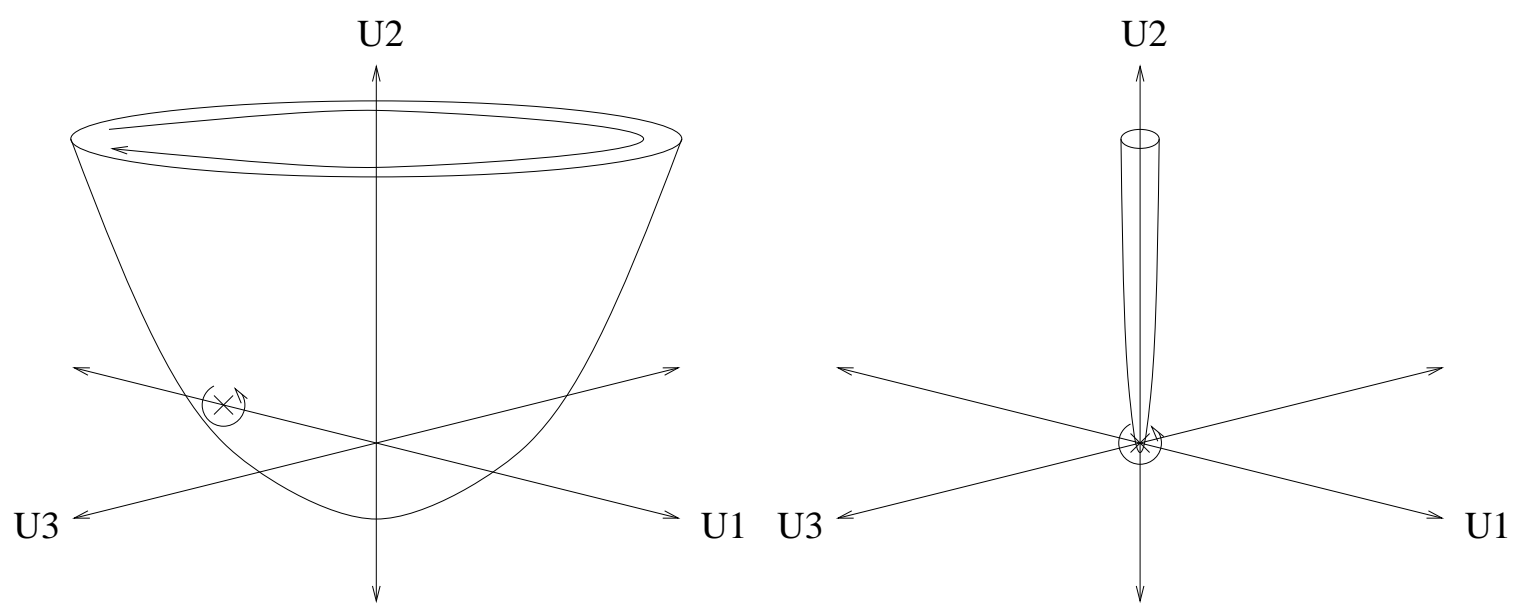

Figure 1: Configuration of branes embedded according to $(v-a w)^{N}=b^{N}$ in the natural coordinates of the near horizon D6-brane geometry. Here, $a=1$ indicated by the fact that the paraboloid points along the $U_{2}$ direction. As $b \rightarrow 0$, the paraboloid degenerates to a "string" like geometry.

$z$ around $a$, and $w$ winds once in the opposite direction if one winds $z$ around $\infty$. The points $z=a$ and $z=\infty$ corresponds to the point at infinity and $\theta=\pi$, respectively, on the paraboloid. Since winding once in $w$ corresponds to winding $N$ times around $x_{11}$, we find that the paraboloid winds $N$ times around circle of 11-th dimension around these two points (See figure 1).

The effects of adjusting parameters $a$ and $b$ are easy to understand. The parameter $a$ controls the orientation of the paraboloid whereas the parameter $b$ controls the eccentricity. For a generic value of $a$, the parabola degenerates into a thin tube as we send $b$ to zero. In this limit, the point at $\theta=\pi$ also approaches the origin, and we recover the configuration of D4-brane ending on the core of D6-brane as in the previous section. The case where $a=\infty$ (keeping $b / a$ finite) appears to be an exception of this general behavior. In this case, the axis of the parabola also points along $\theta=\pi$ direction. This will cause the zero and the pole in the $z$ plane to merge, resulting in a configuration that does not wrap the 11-th direction. Taking $b / a$ to zero will also degenerate the paraboloid into a tube, but it will not wind around the 11-th direction and should not be thought of as a D4-brane. Instead, it a highly curved configuration of NS5-brane stabilized by the gravitational background of the D6-brane.

What we are finding is the point $b=0$ and $a=\infty$ is a special point in the modulispace of curves $(v-a w)^{N}=b^{N}$. The moduli-space of brane configurations with different topology of winding around the 11-th direction is meeting at this point. This structure is strongly reminiscent of the Hanany-Witten transition [3, 21]. 


\subsection{Supergravity Solution}

Now that we understand the geometry associated with this embedding, let us discuss the corresponding supergravity solution. Although the procedure is very straightforward extension of the result of [18] and the previous section, the explicit results are rather lengthy and cumbersome. We will therefore present only schematic results in this section. Enough details will be present so that explicit results, if desired, can easily be reproduced.

The curve (3.1) describes $N$ parallel M5-branes in $R^{1,10}$. This will naturally separate the $(v, w)$ plane into components parallel and transverse to these branes:

$$
z_{\|}=\frac{1}{\sqrt{1+|a|^{2}}}(w+\bar{a} v), \quad z_{\perp}=\frac{1}{\sqrt{1+|a|^{2}}}(v-a w)
$$

The supergravity solution describing these $N$ sets of M5-branes is

$$
\begin{aligned}
d s_{11}^{2} & =f^{-1 / 3}\left(-d x_{0}^{2}+d x_{1}^{2}+d x_{2}^{2}+d x_{3}^{2}+d z_{\|} d \bar{z}_{\|}\right)+f^{2 / 3}\left(d x_{4}^{2}+d x_{5}^{2}+d x_{6}^{2}+d z_{\perp} d \bar{z}_{\perp}\right) \\
A^{(6)} & =\frac{f-1}{f} d x_{0} \wedge d x_{1} \wedge d x_{2} \wedge d x_{3} \wedge\left(\frac{d z_{\|} \wedge d \bar{z}_{\|}}{2 i}\right)
\end{aligned}
$$

where the harmonic function $f$ encodes the fact that there are $N$ sets of M5-branes

$$
f=1+\sum_{k} \frac{\pi n l_{p}^{3}}{r_{k}^{3}}, \quad r_{k}^{2}=x_{4}^{2}+x_{5}^{2}+x_{6}^{2}+\left|z-b e^{2 \pi i k / N}\right|^{2} .
$$

All that remains to be done is to follow the change of variables (2.1) and (2.2) and to dimensionally reduce. The only subtlety that arises for this general case is the fact that the harmonic function $f$ can have explicit dependence on $x_{11}$. This means that background massive Kaluza-Klein fields are generated when (3.4) is dimensionally reduced. These massive Kaluza-Klein excitations can be eliminated if, instead of localizing the M5-branes in the original $R^{1,10}$ space along a polygon, we smear along the circle of radius $|b|$. This will change the form of the harmonic function from (3.5) to

$$
f=1+N \int_{0}^{1} d q \frac{\pi n l_{p}^{3}}{\left(x_{4}^{2}+x_{5}^{2}+x_{6}^{2}+\left|z-b e^{2 \pi i q}\right|^{2}\right)^{3 / 2}} .
$$

Of course, to leading order in large $N$, the background is already smeared and this extra step is not necessary.

Since these backgrounds describe coexisting D4-branes and NS5-branes in the near horizon region of the D6-brane, it would be interesting to examine the behavior of RR 5-form and NSNS 6-form (Poincare dual of NSNS 2-form) in this background. Here, we 
seem to encounter a small puzzle regarding these backgrounds. Let us illustrate this with some examples.

First, consider sending a to zero so that we have a curve $v^{N}=b^{N}$. If we also set $b$ to zero, this reduces to $N$-fold copy of the situation discussed in the previous section. There, we found that only the RR 5-form background and no NSNS 6-form background was generated, which is appropriate for a background consisting only of D4-branes and D6-branes. The fact that only RR 5-form follows from the fact that the M-theory 6-form (3.4) contains a factor

$$
\left(\frac{d z_{\|} \wedge d \bar{z}_{\|}}{2 i}\right)=d x_{7} \wedge d x_{10}=\frac{l_{p}^{3}(1+\cos (\theta))}{4 R_{11}} d U \wedge d x_{11}-\frac{l_{p}^{3} U \sin (\theta)}{4 R_{11}} d \theta \wedge d x_{11},
$$

which is to say that the factor of $d x_{11}$ is always present. Therefore, this 6-form was guaranteed to reduce to a 5 -form in type IIA.

Although this was perfectly fine for $v^{N}=0$, this is somewhat troublesome for $v^{N}=b^{N}$. For by increasing $b$ to non-zero values, what used to be a string-like object (tensored with $R^{1,3}$ ) blows up into a paraboloid (times $R^{1,3}$ ). It would seem such an object is better described as NS5-brane, and we would expect NSNS 6-form to turn on in this background.

On the other hand, $d z_{\|}$does not change under change in $b$ (3.6). Only the form of the harmonic function $f$ changes with changes in $b$. Therefore, the 6 -form in 11 dimensions will always have a $d x_{11}$ component, and the 6 -form will always reduce to RR 5 -form in type IIA.

Similar paradox appears for other configurations. For example, if we consider the curve in the $a \rightarrow \infty$ limit $w^{N}=b^{N}$, where the brane is no longer wrapping the circle in the 11-th dimension, we expect not to have RR 5 -form in the background. It can be easily checked, however, that

$$
d x_{8} \wedge d x_{9}=\frac{l_{p}^{3}(1-\cos (\theta))}{4 R_{11}} d U \wedge\left(d x_{11}+N R_{11} d \psi\right)+\frac{l_{p}^{3} U \sin (\theta)}{4 R_{11}} d \theta \wedge\left(d x_{11}+N R_{11} d \psi\right) .
$$

This time, the M-theory 6-form has components containing and not containing the factor of $d x_{11}$. This means that when reduced to type IIA, both RR 5-form and NSNS 6-form is excited.

Finally, consider the embedding $v-a w=0$. We showed that this embedding describes a parabola that collapsed down to a thin tube, and should be thought of as a D4-brane stretching along $a=\tan (\theta / 2) e^{i \psi}$ and ending on the D6-brane core. However, $\frac{1}{2 i} d x_{\|} \wedge d \bar{z}_{\|}$ will be some linear combination of (3.7) and (3.8), indicating that both NSNS 6-form and the RR 5-form background is turned on. 
It is not clear if this apparent mismatch between the "identity" of the branes and the differential forms in the background is really a problem. Perhaps there is no clear distinction between NS5-branes and D4-branes in the near horizon region of a D6-brane.

\section{Discussions}

The main goal of this paper was to demonstrate that a certain supergravity solution for locally intersecting branes can be derived by taking a flat configuration of M-brane in $R^{1,10}$ and acting by quotient group and dimensionally reducing to type IIA. As a concrete example, we derived the supergravity background for D4-brane ending on a D6-brane, albeit only in the near horizon limit of the D6-brane background. This configuration is precisely that of a D4-brane BIon ending on the D6-brane. Unfortunately, the near horizon geometry of the D6-brane does not in any way correspond to a "decoupling limit" of some world volume field theory [19, 22], so the supergravity solution we derived in section 2 are not expected to capture any aspect of magnetic monopole solution of $6+1$ dimensional gauge theory (whatever that may be). Nonetheless, for small $g_{s t}$ and $N$, one can consider the full Born-Infeld dynamics of the D6-brane $[4,5]$, and infer the existence of a D4-brane BIon state. One of the main observation from the earlier work on BIons is the fact that the branes bend in a particular way to preserve the BPS condition. It would be very interesting to compare aspects of such a state to our supergravity configuration valid at large $N$. The fact that these configurations are BPS should allow some concrete comparison.

Since the directions shared by the D4-brane and D6-brane are isometrics, one can easily T-dualize along these directions. This will give us a localized intersection of D1brane on D3-brane. Near horizon geometry of such a configuration will be of great interest since the gauge theory on D3-brane does decouple in the near horizon limit, and technique similar to ones employed in [17] should allow a direct comparison between supergravity solution and some aspect of the Prasad-Sommerfield magnetic monopole. In fact, the T-dualizing along the $(1,2,3)$ direction leads to dramatic simplification in the form of the dilaton background

$$
\frac{1}{g_{s t}} e^{\phi}=\sqrt{f}
$$

which is closely related to the fact that in the absence of D1-brane, the dilaton background is constant. Unfortunately, T-duality for supergravity solution is only capable of recovering solutions smeared along the dual circle. Furthermore, decoupling and smearing are non-commutative operation. Therefore, we can not expect to recover any informa- 
tion about $3+1$ dimensional Prasad-Sommerfield monopole directly from our supergravity solution.

We also discussed a general class $^{1}$ of M5-branes embedding along a curve $(v-a w)^{N}=$ $b^{N}$ which admits an analogous construction. We showed that in general, these curves describe a paraboloid in the near-horizon geometry of the D6-brane tensored with flat 3+1dimensional worldvolume embedded along the longitudinal coordinates of the D6-brane. In a certain limit, this paraboloid degenerates in to a "string" like configuration terminated at the core of the D6-brane. In a certain sense, the paraboloid can be interpreted as an NS5-brane and its degeneration into string as a D4-brane. However, the background NSNS 6-form and RR 5-form do not to match this identification. It is possible that such an identification is only valid in the asymptotically flat region of space-time and is inapplicable in the near horizon region.

The results reported in this paper are far from solving the long-standing problem of the spacetime background for all allowed brane intersections and junctions. Nonetheless, it is our hope that having explicit solution at our disposal, albeit in a very special case, will provide some useful hint or at least point in the right direction for future work.

\section{Acknowledgments}

It is a pleasure to thank Arkady Tseytlin for correspondence and illuminating discussions. I am also grateful to Ofer Aharony, Per Berglund, Eric D'Hoker, Sunny Itzhaki, and Shamit Kachru for enlightening discussions. This work was supported in part by the National Science Foundation under Grant No. PHY94-07194.

\section{References}

[1] G. T. Horowitz and A. Strominger, "Black strings and p-branes," Nucl. Phys. B360 (1991) 197-209.

[2] J. Polchinski, "TASI lectures on D-branes," hep-th/9611050.

[3] A. Giveon and D. Kutasov, "Brane dynamics and gauge theory," hep-th/9802067.

[4] C. G. Callan and J. M. Maldacena, "Brane Dynamics From the Born-Infeld Action," Nucl. Phys. B513 (1998) 198, hep-th/9708147.

\footnotetext{
${ }^{1}$ Similar idea can be used for branes intersecting Melvin flux tubes. See [23].
} 
[5] G. W. Gibbons, "Born-Infeld particles and Dirichlet p-branes," Nucl. Phys. B514 (1998) 603, hep-th/9709027.

[6] O. Aharony, A. Hanany, and B. Kol, "Webs of $(p, q)$ five-branes, five-dimensional field theories and grid diagrams," J. High Energy Phys. 01 (1998) 002, hep-th/9710116.

[7] K. Dasgupta and S. Mukhi, "BPS nature of three string junctions," Phys. Lett. B423 (1998) 261, hep-th/9711094.

[8] A. Sen, "String network," hep-th/9711130.

[9] S. M. Carroll and M. Trodden, "Dirichlet topological defects," Phys. Rev. D57 (1998) 5189-5194, hep-th/9711099.

[10] J. Maldacena, "The Large N limit of superconformal field theories and supergravity," Adv. Theor. Math. Phys. 2 (1998) 231, hep-th/9711200.

[11] A. A. Tseytlin, "Extreme dyonic black holes in string theory," Mod. Phys. Lett. A11 (1996) 689-714, hep-th/9601177.

[12] A. A. Tseytlin, "Composite BPS configurations of $p$-branes in ten-dimensions and eleven-dimensions," Class. Quant. Grav. 14 (1997) 2085, hep-th/9702163.

[13] R. R. Khuri, "Remark on string solitons," Phys. Rev. D48 (1993) 2947.

[14] J. P. Gauntlett, D. A. Kastor, and J. Traschen, "Overlapping branes in M theory," Nucl. Phys. B478 (1996) 544-560, hep-th/9604179.

[15] J. P. Gauntlett, G. W. Gibbons, G. Papadopoulos, and P. K. Townsend, "Hyper-Kahler manifolds and multiply intersecting branes," Nucl. Phys. B500 (1997) 133, hep-th/9702202.

[16] C.-S. Chu, P.-M. Ho, and Y.-Y. Wu, "D instanton in $\operatorname{AdS}(5)$ and instanton in $\operatorname{SYM}(4), "$ hep-th/9806103.

[17] V. Balasubramanian, P. Kraus, A. Lawrence, and S. P. Trivedi, "Holographic probes of anti-de Sitter space-times," hep-th/9808017.

[18] N. Itzhaki, A. A. Tseytlin, and S. Yankielowicz, "Supergravity solutions for branes localized within branes," Phys. Lett. B432 (1998) 298-304, hep-th/9803103. 
[19] N. Itzhaki, J. M. Maldacena, J. Sonnenschein, and S. Yankielowicz, "Supergravity and the large N limit of theories with sixteen supercharges," Phys. Rev. D58 (1998) 046004, hep-th/9802042.

[20] T. Nakatsu, K. Ohta, T. Yokono, and Y. Yoshida, "A Proof of brane creation via M theory," Mod. Phys. Lett. A13 (1998) 293, hep-th/9711117.

[21] S. Elitzur, A. Giveon, and D. Kutasov, "Branes and N=1 duality in string theory," Phys. Lett. B400 (1997) 269-274, hep-th/9702014.

[22] N. Seiberg, "Why is the matrix model correct?," Phys. Rev. Lett. 79 (1997) 3577-3580, hep-th/9710009.

[23] J. G. Russo and A. A. Tseytlin, "Green-Schwarz superstring action in a curved magnetic Ramond-Ramond background," J. High Energy Phys. 04 (1998) 014, hep-th/9804076. 